\title{
Preliminary study on the application of PspA as carrier
}

Lichan-Wang\#, Yajun-Tan\#, Chen-Wei, Huajie-Zhang, Peng-Luo, Shumin-Zhang*, Xiao-Ma*

DTaP and toxins division, National Institutes for Food and Drug Control, Key Laboratory of the Ministry of Health for Research on Quality and Standardization of Biotech Products, Beijing 10050, China

Abbreviated Names: Wang LC, Tan YJ, Wei C, Zhang HJ, Xu YH, Luo P, Zhang SM, Ma X

Corresponding author: Shumin-Zhang, zhangsm@nicpbp.org.cn; Xiao-Ma, maxia0421@sina.cn \#: These authors contributed equal to this work.

This research was provided financial support by National high-tech research and development plan (project 863), project number: 2012AA02A402.

Abbreviations: PspA, pneumococcal surface protein A; GAMP, group A meningococcal polysaccharide; ELISA, enzyme linked immune sorbent assay; FCM, flow cytometry; SFC, spot forming cells; SBA, serum bactericidal assay; Th, helper T cell; TI, thymus independent; TD, thymus dependent; NIFDC, national institutes for food and drug control; GMT, geometric mean antibody titers; IFN- $\gamma$, fibronectin $\gamma$; IL-4, interleukin -4; ANOVA, one-way analysis of variance

Keywords: pneumococcal surface protein A; group A meningococcal polysaccharide; conjugate; carrier protein; immune effect

\section{Abstract}

The aim of the study is to research the feasibility of pneumococcal surface protein A (PspA) using as carrier protein. Three recombinant pneumococcal surface proteins A (come from family1 and family 2) were expressed by prokaryotic expression system and were conjugated to group A meningococcal polysaccharide (GAMP) to make three polysaccharide-protein conjugates. The conjugates, un-conjugated proteins, GAMP and GAMP-TT vaccine bulk (used as positive control) were immunized to mice and their immune effects were evaluated by the method of ELISA, FCM and SBA. The results showed that the polysaccharide-protein conjugates can produce higher levels of 
anti-GAMP IgG titers $(\mathrm{P}<0.05)$, higher ratios of Th1/Th2 $(\mathrm{P}<0.05)$ and higher levels of serum bactericidal activity $(\mathrm{P}<0.05)$ compared with the un-conjugated GAMP. The conjugation of PspAs to GAMP also enhanced the anti-PspA responses compared with un-conjugated PspAs except PspA3. In conclusion, all the results indicated that three PspAs were suitable carrier protein as demonstrated by the characteristics of a T-cell dependent response to the GAMP, and would protect against group A of epidemic cerebrospinal meningitis and also have the potential to provide broad protection from Streptococcus pneumonia.

\section{Introduction}

Invasive bacteria, such as Haemophilus influenza type b (Hib), Neisseria meningitides and Streptococcus pneumonia, the most important antigen is polysaccharide on the surface of them. Capsular polysaccharide is thymus independent antigen; immunization of infants and young children with these antigens does not induce well and long-lasting protective levels of serum antibodies. The success of Hib glycol-conjugate vaccine highlighted the advantages of converting polysaccharides into T-dependent antigens by chemical conjugation to carrier proteins. Tetanus toxoid, diphtheria toxoid and the non-toxic mutant of diphtheria toxin, CRM197 are commonly used as protein carrier. With the increasing of polysaccharide conjugate vaccines, the development of new protein carrier is necessary for avoiding immune 
tolerance, immune interference or immune suppression due to repeated using the same carrier $(1,2)$. In addition, the imperfection of the polysaccharide and conjugate vaccine is that the protection of them can not cover all serotypes of the bacteria strains. Serotype conversion limits their development and decreases their immune protecting coverage. Thus increasing the selection of carrier protein or developing the new conjugate vaccine without serotype limitation is important and necessary in future.

In order to solve the above problems, we chose pneumococcal surface protein A (PspA) as research subject. PspA is virulence associated protein found on the surface of all clinical isolates of S. pneumonia (3) and interferes with opsonophagocytosis by interfering with complement deposition on the bacterial surface $(4,5)$. It has five domains: a single peptide, $\alpha$-helical and charged $\mathrm{N}$-terminal domain, a proline-rich region, a choline-binding domain and a short hydrophobic tail. PspA is relatively variable at the DNA and protein sequence levels. According to the sequences of $\alpha$-helical region, PspAs have been divided into six clades, which belong to three families $(6,7)$. PspA family 1 contains two clades (1 and 2), PspA family 2 contains three clades (3, 4 and 5) and PspA family 3 contains one clade (clade 6). Families 1 and 2 are expressed in more than 90 percent of strains (8). Antibodies from different clades of the same family have relatively high cross reactivity and cross protection, 
but lower about clades from different families. Based on its structural diversity, it has been suggested that PspA-based vaccine should contain at least one clade from each of the two major families in order to elicit broad protection (9).

In our study, different clades of recombinant PspA protein (clade1, clade2 and clade3) were prepared and conjugated to group A meningococcal polysaccharide (GAMP) as carrier protein to make available a compounds, which not only prevent epidemic cerebrospinal meningitis but also has the potential to provide protection from Streptococcus pneumonia. The immune effects, including humoral and cellular immune responses were assessed after being immune to mice. In this article, we focused on the ability of the conjugates to prevent meningococcal infection, and pneumococcal infection will be covered in a follow-up study.

\section{Materials and methods}

\subsection{Preparation of recombinant PspA proteins}

PspA gene sequences of bacteria strain DBL6A (GenBank AF071805.1）, RX1（GenBank U89711.1） and EF3296（GenBank AF071816.1) were obtained from GenBank and synthesized after being codon optimization in accordance with Escherichia coli codon preference so as to enhance their protein expression in prokaryotic expression system. The synthesized DNAs were 1143bp for PspA/ 

belong to clade 1 , clade 2 and clade 3 respectively. Signal peptide sequences of these DNAs have been excluded. The constructed genes were cloned into plasmid pET-30a $(+)$ vector with Nde I and Xho I restriction enzyme cutting site. PCR and enzyme digestion were used to identify the correction of linkage. The expression plasmid was transformed into competent E.coli BL21 (DE3) and expressed by induction with isopropyl-D-1-thiogalactopyranoside (IPTG). After being purified by several steps of chromatography (GE healthcare), with recombinant PspA by carbodiimide method and purification of the compound with SephacrylS-400 column chromatography. The ratio 
between protein PspA and GAMP was determined by improved Lowry method and Phosphorus assay respectively (10). Table 1 is the results of the conjugates. GAMP-TT (used as positive control) is vaccine bulk provided by division of respiratory tract bacterial vaccine of NIFDC.

\subsection{Animal immunization}

Groups of Blab/C female mice weighting 16-18g were injected subcutaneously with each of the conjugate preparations or unconjugated controls. The dose of polysaccharide per injection was fixed at $2.5 \mu \mathrm{g}$. The dose of protein varied from conjugate to conjugate and is presented in table 1. Unconjugated proteins as control were injected $5 \mu \mathrm{g}$ per mice. Mice received 3 doses at day0, day 14 and day28 and were bled by retro orbital puncture at day13, day27, day35 and day180. Serum were separated and stored at $-20^{\circ} \mathrm{C}$ for testing. All animal experiments were accordance with Regulations on Management of Laboratory Animal (Ministry of Science and Technology) 1988, China.

\subsection{Antibody detection}

Anti-PspA and anti-GAMP IgG titers at day13, day27, day35 and day180 were assayed respectively by enzyme-linked immune sorbent

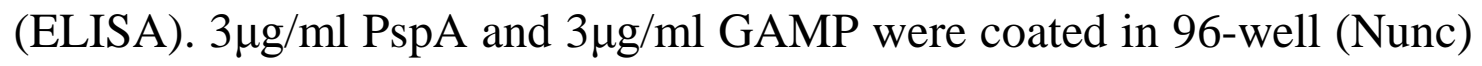
plates. Sera were 2 fold serially diluted across the plates with the predetermined initial dilution. HRP-conjugated goat anti-mouse IgG (Santa Cruz) and OPD (Ameresco, USA) substrate were used as the 
second antibody and color agent. The reaction was stopped by adding $50 \mu \mathrm{l}$ of $1 \mathrm{M} \mathrm{H} 2 \mathrm{SO} 4$ and was read at $490 \mathrm{~nm}$ with microplate reader.

\subsection{Th1/Th2 detection by FCM}

Seven days after the third immunization (days 35), $1 \mathrm{ml}$ spleen cells $\left(2 \times 10^{6} / \mathrm{mL}\right)$ from mice of each group were co-cultured with $50 \mathrm{ng} / \mathrm{ml}$ PMA(sigma), $1 \mu \mathrm{g} / \mathrm{ml}$ ionomycin (Sigma) and 10 $\mu \mathrm{g} / \mathrm{ml}$ BFA(Biolegend) in their final concentration for $4-6$ hours at $37^{\circ} \mathrm{C}$ with $5 \% \mathrm{CO}_{2}$ incubator. Washing cells with cell staining buffer and labeling with mouse CD3-PerCP, CD4-FITC antibody (Biolegend) for 20mins in the dark. Then after the cells were fixed with fixation buffer and permeabilization with permeabilization wash buffer, which were labeled with mouse IFN- $\gamma$ PE and IL-4APC antibody (Biolegend) for 30mins in the dark and then were washed and suspended in cell staining buffer for FCM analysis.

\subsection{Bactericidal detection}

Serum bactericidal assay (SBA) is one of the best methods on assessing the potency of meningococcal polysaccharide vaccine, which mainly detect the functional antibodies contained in serum. Meningococcal strain and baby rabbit complement were provided by Lanzhou institute of biological products. Serum samples and rabbit complement for control were heat inactivated for $30 \mathrm{mins}$ at $56^{\circ} \mathrm{C}$ for use in the assay. Make serum sample and control sample to 3 fold serial dilutions for a total of 8 dilutions in 96 well $\mathrm{U}$ micro titre plates with 
166

167

initial 4 fold dilution. Add $10 \mu$ l of group A meningococcal strain to each well and gently tap plates to mix for 30mins, then add $10 \mu 1$ of complement or heat inactivated complement to control well and sample well respectively, which cultured for $1 \mathrm{hr}$ at $37^{\circ} \mathrm{C}$ with $5 \% \mathrm{CO}_{2}$ incubator. Mix plate and take $10 \mu \mathrm{l}$ from eight serial diluted well onto the THYA plate and tilt plate immediately, make the liquid flow into one, about 2-3 $\mathrm{cm}$. Other samples and so on. The plates were placed in room temperature for $20 \mathrm{mins}$ and transfer to $37^{\circ} \mathrm{C}$ with $5 \% \mathrm{CO}_{2}$ incubator for overnight. After overnight incubation, THYA plates were covered with TTC superstardom agar and using automatic colony counter count colony number after agar solidification. According to the control panel of colony, counting experimental group 50\% sterilization drops.

\subsection{Statistical analysis}

IBM SPSS Statistics 20 was used to analysis. The significance of differences in different groups was assessed using a one-way analysis of variance (ANOVA). Pearson correlation was used to analyze the correlation between the results of SBA and antibodies. Kolmogorov-Smirnov was used to test the normality of data. For all comparisons, a P-value of $<0.05$ was considered to represent statistical significance.

\section{Results}

\subsection{Production and purification of PspAs}


The amplified products were 1000 to 2500 base pairs (bp) by PCR and enzyme digestion using expression plasmids as template, which are

190 consistent with the expected size (figure1 A to B). The three proteins 191 were soluble expressed by Escherichia coli induced with IPTG(final

192

concentration $1 \mathrm{mmol} / \mathrm{L}$ ) at $37^{\circ} \mathrm{C}$ for $4 \mathrm{hrs}$, which about $30 \%$ to $40 \%$ of the amount of total expression. Through three steps of chromatography, the purity of them reached 90 percent (figure1 D to F). Western bot results (figure1 C) showed that the three proteins can react with human serum of clinically diagnosed pneumonia, which indicated the proteins have good antigenicity.

\subsection{Chemical and physical analysis of the conjugates}

Three conjugates were synthesized: PspA1-GAMP, PspA2-GAMP and PspA3-GAMP, prepared with cyanogen bromide activation method. The content of protein and polysaccharide were tested by the methods described in Chinese pharmacopoeia (10). The ratio of polysaccharide and protein were calculated according to their concentration. Table 1 shows the results.

\subsection{Immunogenicity of the conjugates and un-conjugates}

The anti-GAMP, anti-PspA and anti-TT titers were calculated through compared with that of PBS control mice sera, and expressed as the reciprocal of geometric mean titers (GMT). The anti-GAMP responses are presented in figure 2. All conjugates induced anti-GAMP 
responses after one dose, and increased with the second and third booster immunization, which were significantly higher than that of unconjugated GAMP, $\mathrm{P}<0.05$. The anti-PspA (three clades) and anti-TT responses are presented in table 2. The dose of PspA was almost same for each conjugate due to the ratio of GAMP and PspA is similar. The anti-PspA1 and anti-PspA2 responses following the first dose of conjugate were low; responses were all boosted after the second and third dose, which were significantly higher than that of unconjugated PspA1 and PspA2, $\mathrm{P}<0.05$. The anti-PspA3 titer to conjugates with PspA3 carrier protein was significantly lower after the third dose than that of unconjugated PspA3, $\mathrm{P}<0.05$. For GAMP-TT conjugate, anti-TT titer was almost dozens of times lower compared to that of unconjugated TT either after the first immunization or after the booster immunizations, which indicates the conjugates inhibited the immunogenicity of TT.

\subsection{FCM results of the conjugates and un-conjugates}

The test mainly detected the condition of immunized mice spleen cells after stimulating with non-specific stimulants in vitro. IFN- $\gamma$ and IL-4 antibody were used as markers for Th1 and Th2 cell groups. The results of Th1/Th2 ratio were showed in figure3. Compared with un-conjugated GAMP and PBS control, the ratio of Th1/Th2 in groups of polysaccharide-protein conjugates were significantly higher, $\mathrm{P}<0.05$.

The ratio in the three GAMP-PspA conjugates was similar, $\mathrm{P}>0.05$, 
which were higher than that of in GAMP- TT conjugates, $\mathrm{P}<0.05$. There were no significant differences between the three un-conjugated PspA groups and the three GAMP- PspA conjugates, $\mathrm{P}>0.05$.

\subsection{Bactericidal activity}

Serum bactericidal assay is the most convincingly test, which can directly reflect the potency of meningococcal vaccine. SBA activities were expressed by geometric mean antibody titer. Figure 4 showed the results of test. Anti-GAMP IgG antibody titers in each groups were also showed in the figure, the trend of which were nearly the same with that of SBA results. Pearson correlation was used to analyze the correlation between the results of SBA and antibodies, which indicated the SBA activity is positive correlation with the antibody levels of anti-GAMP, $\mathrm{R}=0.604, \mathrm{P}<0.05$. The polysaccharide-protein conjugates have higher SBA activity compared with that of un-conjugated GAMP, the difference is significantly, $\mathrm{P}<0.05$.

\section{Discussion}

According to the genome sequences of corresponding protein in GenBank, we adopt the method of chemical synthesis for our targeting genes. This method can omit the bacterial culture process, and can also obtain any kind of bacteria gene sequences that can be found in GenBank. At the same time, codon optimization before the gene sequence synthesis can improve the expression level of target protein. In our study, we get 
254

gene sequences of PspA proteins for streptococcus pneumonia strains DBL6A, RX1 and EF3296 from GenBank. The gene sequences were synthesized after being codon optimization, and connected to prokaryotic expression vector PET30a. it is confirmed by sequencing that the correctness of the connection, and the target gene fragment is in line with corresponding sequences in GenBank and the sequence of amino acids have no mutations. The three PspA proteins were highly expressed with soluble formulation, which account for about 30\%-40\% in total bacterial proteins respectively. After being purification by three steps of chromatography, the purity of them reached 90 percent. The proteins were identified by western blot with human serum of clinically diagnosed pneumonia.

Bacteria capsular polysaccharide is TI antigen, which cannot stimulate $\mathrm{T}$ cells to produce cellular immune responses and cannot produce immune memory. Therefore, the researcher combines carrier protein and polysaccharide by chemical method, which changes the TI antigen properties of polysaccharides for TD antigen and solved the above questions (11). PspA was selected as carrier protein of GAMP conjugate for its potential to provide broad cross-reactive protection against S. pneumonia. The ratio of GAMP: PspA is about 0.63 , which is similar for families 1 and 2. 
276 suitable carrier proteins for preparation of GAMP conjugates.

277 Conjugation of PspA to GAMP resulted in a T-cell dependent response to

278 the GAMP as demonstrated by boosting the antibody responses following

279 the second dose and the third dose, which means the induction of 280 immunological memory. Anti-GAMP responses obtained after 3 doses of 281 the GAMP-PspA conjugates were similar to those achieved with GAMP-TT conjugates. Un-conjugated PspA from family 1 was poorly immunogenic compared with that of conjugation of PspA to GAMP, which means the conjugation significantly increased the anti-PspA response. However, the conjugation of PspA from family 2 to GAMP did not increase the level of anti-PspA antibody, which was lower than that of un-conjugated PspA3, $\mathrm{P}<0.05$, especially after the third dose. The anti-TT response for GAMP-TT is similar to GAMP-PspA3; the immunogenicity of TT was inhibited by the conjugation to GAMP. This

290 may be due to the coverage of protein antigen epitope or due to the 291 protein changes in conformation in the process of combining 292 polysaccharide to protein $(12,13)$. For TT, another possibility is that the destruction of T cell epitope in the chemical detoxification process, which 294 affecting its immunogenicity (14). The exact cause remains to be 295 confirmed. 
used. For Th1/Th2 ratio detected by FCM, compared with un-conjugated GAMP and PBS control, the ratio in groups of conjugates were significantly higher, $\mathrm{P}<0.05$. The above results indicated that the carrier protein conjugated with GAMP can activate the cellular immune response.

Serum bactericidal assay is mainly detecting the activity of functional antibody in the serum, which is the most direct method in the evaluation of meningococcal polysaccharide vaccine efficacy. It has proved it is associated with the protection of vaccine in the 1860s (15-18), and it has become the gold standard for evaluation of meningococcal vaccine efficacy. The results of SBA showed that the bactericidal activities of the conjugates were significantly stronger than that of un-conjugated GAMP, and the potency of GAMP-PspA1 was higher than that of GAMP -TT, P < 0.05; other PspA conjugates (PspA2, PspA3) had no statistical differences compared with GAMP-TT group, $\mathrm{P}>0.05$.

In conclusion, described here is a procedure for producing a GAMP conjugates using PspAs as carrier protein, and evaluation of the feasibility of PspA proteins. The method of humoral immune response, cellular immune response and SBA assay were used to verify PspA proteins from both families 1 and 2 were suitable carrier for preparation of GAMP conjugates. The research to a certain extent promotes the development of new carrier protein, and it is also the basis for the 
research of PspA protein as carrier for other kinds of bacterial polysaccharide vaccine. However, there are many areas of study that need to be continued, such as the side effects of PspA and the resistance to pneumococcal infection. It will be studied in detail in subsequent trials.

\section{Acknowledgments}

The authors would like to acknowledge the contributions of Ms. Ruijie Qiao, Mr. Zhiqiang Zhao and Mr. Lin Du, assisting with the test of serum bactericidal assay.

\section{References}

1. Mawas F, Dickinson R, Douglas-Bardsley A, Xing DK, Sesardic D, Corbel MJ. Immune interaction between components of acellular pertussis-diphtheria-tetanus (DTaP) vaccine and Haemophilus influenzae b (Hib) conjugate vaccine in a rat model. Vaccine, 2006, 24:3505-3512.

2. Eva Maria Pollabauer, Robert Petermann, Hartmut J. Ehrlich.The influence of carrier protein on the immunogenicity of simultaneously administered conjugate vaccines in infants. Vaccine, 2009, 27:1674-1679.

3. Briles DE, Tart RC, Swiatlo E, et al. Pneumococcal diversity: considerations for new vaccine strategies with emphasis on pneumococcal surface protein A (PspA).Clin Microbiol Rev JT - Clinical microbiology reviews, 1998, 11(4):645-57.

4. Tu AH, Fulgham RL, McCrory MA, Briles DE, Szalai AJ. Pneumococcal surface protein A inhibits complement activation by Streptococcus pneumoniae. Infect Immun 1999, 67(9):4720-4.

5. Kadioglu A, Weiser JN, Paton JC, Andrew PW. The role of Streptococcus pneumonia virulence factors in host respiratory colonization and disease. Nat Rev Microbiol 2008 ;(6):288-301.

6. McDaniel LS, Sheffield JS, Delucchi P, Briles DE. PspA, a surface protein of Streptococcus pneumoniae, is capable of eliciting protection against pneumococci of more than one capsular type. Infect Immun 1991; 59:222-8.

7. McDaniel LS, Ralph BA, McDanial DO, Briles DE. Localization of protection-eliciting epitopes on PspA of Streptococcus pneumoniae between amino acid residues 192-260. Microb Pathog 1994; 17:323-37. 
8. Hollingshead SK, Becker R, Briles DE. Diversity of PspA: Mosaic genes and evidence for past recombination in Streptococus pneumoniae. Infect Immun 2000; 68(10):5889-5900.

9. Melin MM, Hollingshead SK, Briles DE, Lahdenkari MI, Kilpi TM, Kayhty HM. Development of antibodies to PspA families 1 and 2 in children after exposureto Streptococcus pneumoniae. Clin Vaccine Immunol 2008; 15(10):1529-35.

10. Chinese Pharmacopeia, version 3. 2015 ed, p.80-82.

11. Stein KE. Thymus-independent and thymus-dependent responses to polysaccharide antigens. J Infect Dis 1992; 165(Suppl. 1):S49-52.

12. Dick W E Jr, Beurret M. Glycoconjugate of bacterial carbohydrate antigens. A survey and consideration of design and preparation factors. Contrib Microbiol Immunol. 1989; 10: 48-114.

13. Stein K E. Glycoconjugate vaccines. What next? Int J Technol Assess Health Care. 1994; 10(1): 167-176.

14. Carl E Frash. Preparation of bacterial polysaccharide-protein conjugates: analyt ical and manufacturing challenges. Vaccine, 2009; 27(46): 6468-6470.

15. Goldschneider I, Gotschlich EC, Artenstein MS. Human immunity to the meningococcus. I. The role of humoral antibodies. J Exp Med. 1969; 129(6):1307-1326.

16. Goldschneider I, Gotschlich EC, Artenstein MS. Human immunity to the meningococcus. II. Development of natural immunity. J Exp Med. 1969; 129(6):1327-1348.

17. Gotschlich EC, Goldschneider I, Artenstein MS. Human immunity to the meningococcus. IV. Immunogenicity of group $\mathrm{A}$ and group $\mathrm{C}$ meningococcal polysaccharides in human volunteers. J ExpMed. 1969; 129(6):1367-1384.

18. Gotschlich EC, Goldschneider I, Artenstein MS. Human immunity to the meningococcus. V. The effect of immunization with meningococcal group $\mathrm{C}$ polysaccharide on the carrier state. J Exp Med.1969; 129(6):1385-1395. 


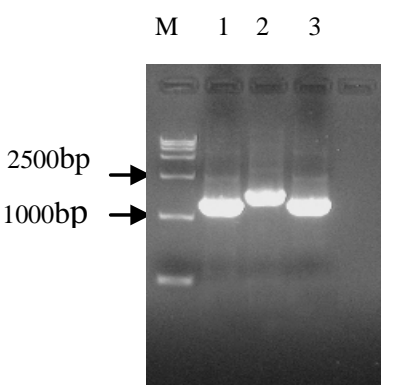

A

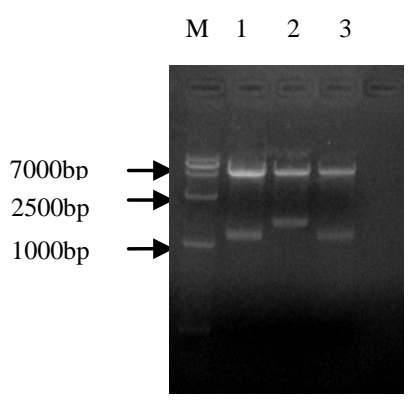

B

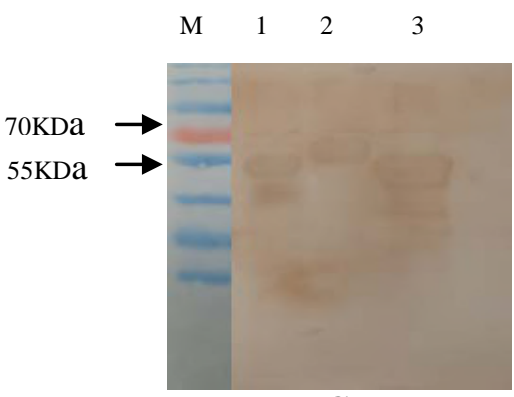

C

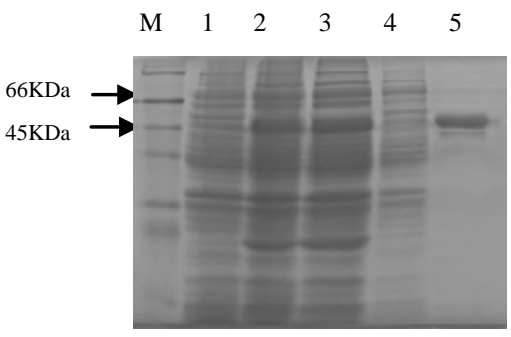

D

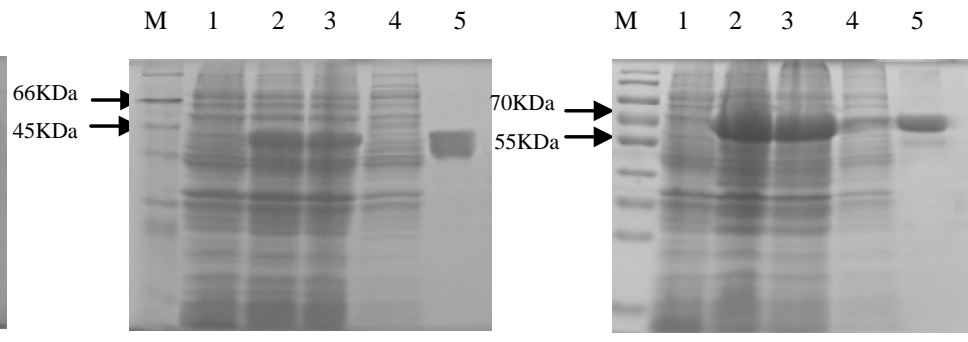

$\mathbf{E}$

$\mathbf{F}$

Figure1. Preparation and identification of three PspA proteins. A to B: Identification results by PCR (A) and enzyme digestion (B) of three PspA expression plasmids. M: DNA Marker, Lane1: PspA1 protein, Lane2: PspA3 protein, Lane3: PspA2 protein. D to F: SDS-PAGE results for recombinant protein PspA1, PspA2 and PspA3. M: Marker; Lane1: empty vector control; Lane2: recombinant protein 4h after induction with IPTG; Lane3: supernatant of recombinant protein after sonication; Lane4: precipitation of recombinant protein after sonication; Lane5: supernatant of recombinant protein after purification. C: Western blot results of PspA proteins. M: Marker; Lane1: recombinant protein PspA1; Lane2: recombinant protein PspA3; Lane3: recombinant protein PspA2. 
410

411

412

413

414

415

416

417

418

419

420

421

422

423

424

425

426

427

428

429

430

431

432

433

434

435

436

437

438

439

440

441

442

443

444

445

446

447

448

449

450

451

452

453

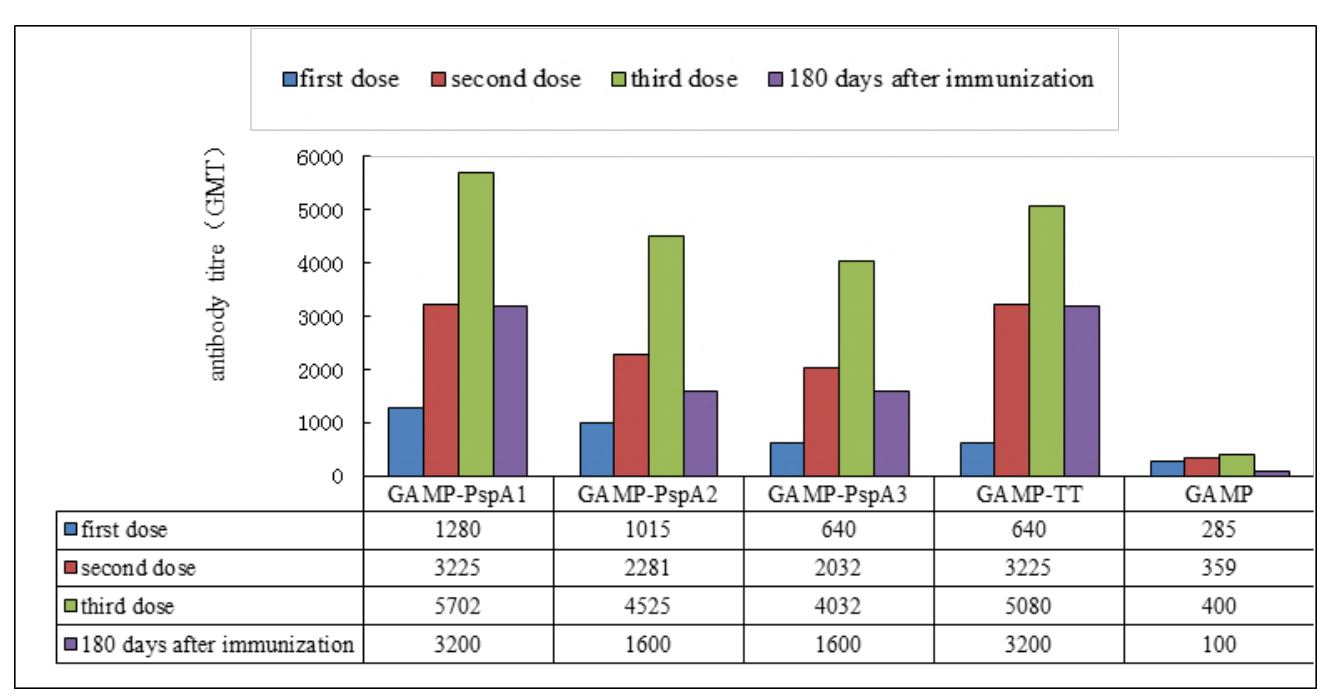

Figure2. Anti-GAMP antibody titers of the conjugates and un-conjugates after the first, second, third dose and 180 days after the first immunization. The results expressed as the reciprocal of geometric mean titers (GMT). 
454

455

456

457

458

459

460

461

462

463

464

465

466

467

468

469

470

471

472

473

474

475

476

477

478

479

480

481

482

483

484

485

486

487

488

489

490

491

492

493

494

495

496

497

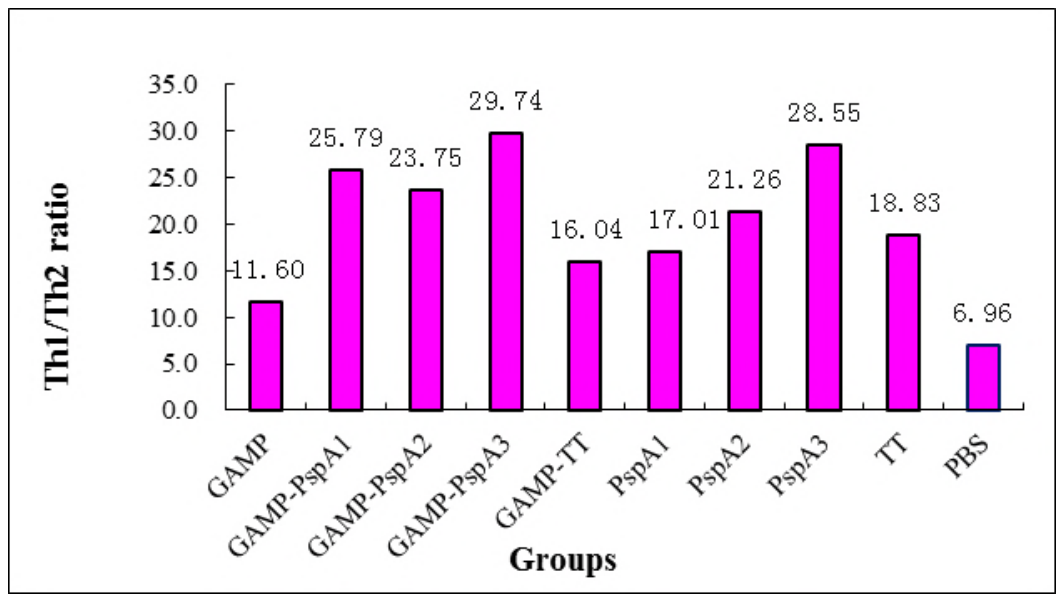

Figure3. Results of FCM: Th1/Th2 ratio of the conjugates and un-conjugates by FCM in mice 7 days after the third immunization. 


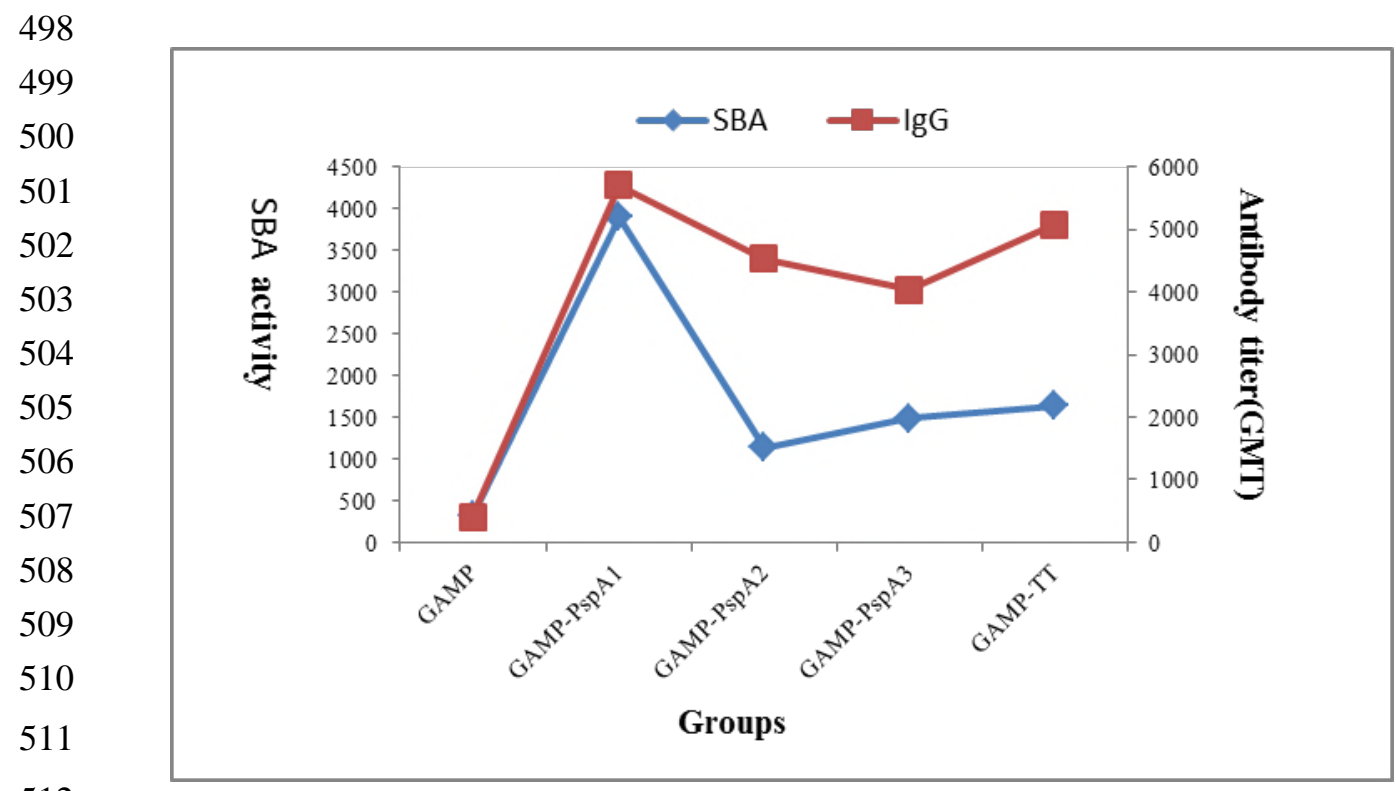

512

514

515

516

517

518

519

520

521

522

523

524

525

526

527

528

529

530

531

532

533

534

535

536

Figure4. SBA activity and anti-GAMP antibody levels of the conjugates and un-conjugates in mice 7 days after the third immunization. There is a consistent trend and positive correlation between the two $(\mathrm{R}=0.604, \mathrm{P}<0.05)$. The antibody results expressed as the reciprocal of geometric mean titers. (GMT).

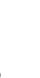


Table1. The ratios of polysaccharide to protein for GAMP-PspA conjugates

\begin{tabular}{lccc}
\hline Groups & $\begin{array}{c}\text { Polysaccharide content } \\
(\mu \mathrm{g} / \mathrm{ml})\end{array}$ & $\begin{array}{c}\text { Protein content } \\
(\mu \mathrm{g} / \mathrm{ml})\end{array}$ & $\begin{array}{c}\text { Ratio of polysaccharide } \\
\text { / protein }\end{array}$ \\
\hline GAMP-PspA1 & 57.3 & 91.4 & 0.63 \\
GAMP-PspA2 & 63.1 & 100.8 & 0.63 \\
GAMP-PspA3 & 121.5 & 195.6 & 0.62 \\
\hline
\end{tabular}

538

539

540

541

542

543

544

545

546

547

548

549

550

551

552

553

554

555

556

557

558

559

560

561

562

563

564

565

566

567

568

569

570 
bioRxiv preprint doi: https://doi.org/10.1101/305102; this version posted April 20, 2018. The copyright holder for this preprint (which was not certified by peer review) is the author/funder. All rights reserved. No reuse allowed without permission.

571

Table 2.Anti-protein IgG antibody levels of the conjugates and un-conjugated proteins after each dose(GMT)

\begin{tabular}{lccc}
\hline Groups & First dose & Second dose & Third dose \\
\hline PspA1 & 45 & 640 & 9051 \\
GAMP-PspA1 & 202 & 1280 & 14368 \\
PspA2 & 113 & 806 & 7352 \\
GAMP-PspA2 & 160 & 2560 & 25600 \\
PspA3 & 403 & 1140 & 51200 \\
GAMP-PspA3 & 202 & 2032 & 7184 \\
TT & 1016 & 10240 & 40637 \\
GAMP-TT & 45 & 113 & 356 \\
\hline
\end{tabular}

572 Note: The results expressed as the reciprocal of geometric mean titers (GMT).

573

574

575

576

577

578

579

580

581

582

583 